\title{
Clinical utility of genetic and genomic services: a position statement of the American College of Medical Genetics and Genomics
}

\author{
ACMG Board of Directors ${ }^{1}$
}

Disclaimer: These recommendations are designed primarily as an educational resource for medical geneticists and other healthcare providers to help them provide quality medical genetics services. Adherence to these recommendations does not necessarily ensure a successful medical outcome. These recommendations should not be considered inclusive of all proper procedures and tests or exclusive of other procedures and tests that are reasonably directed to obtaining the same results. In determining the propriety of any specific procedure or test, geneticists and other clinicians should apply their own professional judgment to the specific clinical circumstances presented by the individual patient or specimen. It may be prudent, however, to document in the patient's record the rationale for any significant deviation from these recommendations.

Genet Med advance online publication 12 March 2015
Clinical utility for genetic tests was discussed in 1998 by the US Task Force on Genetic Testing. The Task Force specifically stated that "the development of tests to predict future disease often precedes the development of interventions to prevent, ameliorate, or cure that disease in those born with genotypes that increase the risk of disease. Even during this therapeutic gap, benefits might accrue from testing." In the broadest sense, "clinical utility" refers to the likelihood that a given intervention (in this case, genetic information) will lead to an improved health outcome ${ }^{2}$ or to whether a test can provide information about diagnosis, treatment, management, or prevention of a disease that will be helpful to a consumer. ${ }^{3}$ Establishing an etiological diagnosis is generally asserted to no longer be sufficient to claim clinical utility. Further, evidence that physicians change their management of a patient based on an etiological diagnosis is said to lack clinical utility unless clinical outcomes research has demonstrated that such changes in an individual's treatment will result in benefit. ${ }^{4}$ Moreover, coverage decision-making policy is now driven by a narrowed perspective that clinical benefit accrues only to the individual receiving the services.

The American College of Medical Genetics and Genomics (ACMG), which represents the medical genetics professional community, profoundly disagrees with the narrow view held by some payers. We submit that the clinical utility of genetic testing and services should take into account effects on diagnostic or therapeutic management, implications for prognosis, health and psychological benefits to patients and their relatives, and economic impact on health-care systems. We believe that clinical utility must also take into account the value a diagnosis can bring to the individual, the family, and society in general.
For example, the diagnosis of a genetic disorder often provides highly significant information to family members who are at risk for such disorders. The assessment of risk to family members of individuals with genetic disorders fits poorly into current models. These define utility as either (i) clinical benefit specific to the individual receiving the service or (ii) "personal" benefit because it applies to other family members or because the result may suggest interventions that are less well defined. ${ }^{5,6}$ Such a construct frames the utility of genetic and genomic information too narrowly and fails to acknowledge that information regarding significant genetic risks can enable highly actionable-indeed, life-saving-interventions for individuals and their family members. Hence, enabling disease avoidance in individuals or families should be considered an overt medical utility rather than as a "personal" utility. As genetic and genomic information increasingly enables disease prevention and reproductive planning, a narrow focus on medical benefit only to the individual originally tested and diagnosed is apparent as a construct of an obsolete system in which care is provided only to those with overt disease and that clinical benefit can be achieved only when a therapeutic option (i.e., a drug) is available. ACMG believes that the interests and lives of family members should be an important clinical consideration in the care of patients. The challenge is in balancing the physician's responsibilities to the patient with the important medical interests of the family.

ACMG believes there is great clinical value in arriving at a precise medical diagnosis, enabling, among other things, identification of a disorder's cause and prognosis, as well as frequently informing preventive and treatment modalities. ACMG

${ }^{1}$ American College of Medical Genetics and Genomics, Bethesda, MD, USA. Correspondence: Michael Watson (mwatson@acmg.net)

Approved by the ACMG Board of Directors, 23 February 2015. 
considers the following to be important clinical utilities related to genetic/genomic information.

\section{CLINICAL UTILITY FOR INDIVIDUAL PATIENTS}

Etiological diagnoses of genetic diseases that are medically actionable with clear benefit to patient outcomes are generally covered by most payers or public health programs. Examples include:

- Situations in which definitive diagnosis specifically informs causality, prognosis, and treatment

- Newborn screening for conditions recommended by the Secretary's Discretionary Advisory Committee on Heritable Disorders of Newborns and Children

- The discovery of medically actionable secondary findings in the course of genomic testing that have associated treatments that improve/affect outcome ${ }^{7}$

Advances in molecular diagnostic technology continue to expand the number of disorders that can be precisely diagnosed through such mechanisms as sequencing or microarrays. While causality is informed in these individuals, prognosis and treatment may not always be available at the present time, as recognized by the US Task Force of Genetic Testing more than 25 years ago. ${ }^{1}$ Arriving at a precise diagnosis always has the potential to lead to a change in medical management, however, even when directly preventing or lessening complications is not possible. For example, an etiologic diagnosis prevents additional unnecessary testing, provides the opportunity for anticipatory guidance, and provides better information regarding recurrence risks for the family and the affected individual. Further, as increased numbers of individuals are diagnosed with specific genetic disorders, information will be obtained that will help predict future complications and risks, tailor medical interventions, and lead to the development of new specific therapies and management strategies. Examples include:

- Patients with complex and often poorly understood clinical disorders such as autism spectrum disorders and intellectual disability

- Patients with rare disorders, including those diagnosed by chromosome analysis (such as karyotype) or microarray

- Patients with genetic conditions such that definitive and specific guidance regarding prognosis and medical management is not yet available

\section{CLINICAL UTILITY FOR FAMILIES}

Definitive diagnosis of a genetic disease

A definitive diagnosis provides the following utility:

- Enables at-risk family members to obtain testing to determine whether they carry a causative mutation, offering the possibility for early intervention.
This clinical utility is independent of whether the affected family member has benefited directly from this diagnosis.

- Enables specific and informed reproductive decisionmaking and family planning.

- Brings resolution to the costly (in terms of both psychosocial and financial contexts) and wasteful (for the medical system at large) diagnostic odyssey that is often pursued in a quest to establish a diagnosis. There are countless examples of economic and psychological costs to the health-care system and to patients and families during the quest to obtain a diagnosis. ${ }^{8}$

- Enables involvement in disease support groups and other types of social support for families.

Not only can genetic testing inform genetic risks in other family members, but testing of other family members can sometimes/often inform the interpretation of results in a patient. For example, information regarding whether a candidate variant is de novo or inherited provides powerful evidence of its potential pathogenicity, thereby giving the finding utility in other family members. Genome-scale testing of parents and a patient (trio testing) also reduces the number of variants that have to be considered as causative, thereby facilitating better interpretation of testing results and minimizing reporting of costly (in terms of both patient well-being and economic terms) false-positive results.

\section{CLINICAL UTILITY FOR SOCIETY}

A specific diagnosis is commonly required for patients to be eligible to participate in clinical trials. The absence of an etiological diagnosis leaves patients without an answer to explain their disorder and transfers to industry or academic institutions and their funders the responsibility to establish disease etiology, further increasing the costs of clinical trials and disenfranchising patients.

- Understanding the etiology of disease and increased accrual into clinical trials will propel research, benefitting society as a whole.

- Many genetic disease risks can be identified decades before the time when benefits accrue to the individual or their family members. In the current health-care environment, cost-effectiveness often is measured by return on investment to payers and is measured over much shorter time periods, despite long-term benefits to population health.

ACMG believes that the nature of inherited genetic disease that can segregate within families of affected individuals provides significant utilities to families and society that are in addition to those benefits that accrue to patients.

\section{DISCLOSURE}

The author declares no conflict of interest. 


\section{REFERENCES}

1. Holtzman NA, Watson MS (eds). Promoting safe and effective genetic testing in the United States. Final report of the Task Force on Genetic Testing. 1997. http:// www.genome.gov/10001733. Accessed 23 November 2014.

2. http://www.phgfoundation.org/tutorials/clinicalUtility/. Accessed 23 November 2014

3. Genetic Home Reference. http://ghr.n/m.nih.gov/handbook/testing/validtest. Accessed 23 November 2014

4. MolDx. Clinical Test Evaluation Process (CTEP). M00096. http://www. palmettogba.com/palmetto/moldx.nsf/MolDX_Manual.pdf. Accessed 22 February 2014
5. Grosse SD, Khoury MJ. What is the clinical utility of genetic testing? Genet Med 2006;8:448-450.

6. Robson ME, Storm CD, Weitzel J, Wollins DS, Offit K; American Society of Clinical Oncology. American Society of Clinical Oncology policy statement update: genetic and genomic testing for cancer susceptibility. J Clin Oncol 2010;28:893-901.

7. Green RC, Berg JS, Grody WW, et al.; American College of Medical Genetics and Genomics. ACMG recommendations for reporting of incidental findings in clinical exome and genome sequencing. Genet Med 2013;15:565-574.

8. Burton H. Ending a diagnostic odyssey. http://www.phgfoundation.org/ blog/16008/. Accessed 23 February 2015. 